\title{
Mass loss and AGB evolution in extra-galactic stellar populations
}

\author{
Albert A. Zijlstra \\ Department of Physics, UMIST, P.O. Box 88, Manchester M60 1QD, \\ $U K$
}

\begin{abstract}
The effect of metallicity on the AGB mass loss is reviewed. Observations have mainly been limited to the Magellanic Clouds but are observationally feasible throughout the Local Group. Expansion velocities are predicted to depend strongly on $Z$ but the peak mass-loss rates appear not to. The Mira $P L$ relation shows no evidence for a $Z$ dependence, giving a powerful potential distance indicator. I derive a distance modulus to the LMC of $m-M=18.63 \pm 0.09$ as well as a bright calibration for the Horizontal Branch in globular clusters. Finally, the predicted initial-final mass relation at low $Z$ is shown to give rise to higher mass remnants. This would result in an increased supernova rate in young, low- $Z$ populations, such as found in the early Universe.
\end{abstract}

\section{Introduction}

AGB mass loss is generally believed to be a two-step process, involving pulsationdriven atmospheric shock waves, followed by radiation pressure on the dust driving the outflow. The dust production rate, and the eventual dust-to-gas ratio of the ejected material, will depend on the metallicity of the star. The details of the mass loss may therefore be different for different stellar populations.

Existing theoretical formalisms for the mass loss are generally derived from the Reimers' relation: $\dot{M}_{R}=-410^{-13} L R / M$. This equation is adjusted to fit existing observations as well as constraints such as the initial-final mass relations. Examples are Blöcker's law $\left(\dot{M}=-4.810^{-9}(L R / M)\left(L^{2.7} / M\right)\right)$, Vassiliadis \& Wood's (1993) formalism and the relation from Judge \& Stencel $\left(\dot{M}=(2.3+/-1.3) 10^{-14}\left(R^{2} / M\right)^{1.43+/-.23}\right)$. Because observations of AGB mass loss have been largely limited to galactic stars, these formalisms have limited predictive power for different stellar populations. So far, only Bowen \& Willson (1992) have attempted to predict mass-loss rates at low $Z$ from theoretical pulsation models.

The study of extra-galactic sources is thus of interest to extend the parameter space of mass loss. At the same time, since the majority of stars with life-times less than the Hubble time will have passed through the AGB, a better understanding of mass loss especially at low $Z$ is also important for our knowledge on stellar remnants and on the recycling of stellar gas into the ISM. The lack of observational data is mainly due to the difficulty to detect dust at any 
large distance, and the paucity of 'dusty' AGB stars due to the short life-time of the superwind phase.

\section{Extra-galactic observations}

\subsection{Capabilities}

Existing and planned instrumentations have the capability to detect AGB stars to distances far outside the Local Group. Observations of mass loss of individual AGB stars is observationally feasible at least for the satellites of the Galaxy. Of these, studies of the Magellanic Clouds have already been initiated (Reid 1991; Wood et al. 1992; Zijlstra et al. 1996; van Loon et al. 1997,1998; Groenewegen \& Blommaert 1998).

Table 1 lists the observational limits of some present and future instrumentation. The last column gives the maximum distance to which a luminous AGB star may be detected given these limits. The K-magnitudes refer to an unobscured, high-mass AGB star with $\mathrm{K}=-9$. The N-band is scaled from an obscured luminous AGB star in the LMC. The NGST value refers to lowresolution spectroscopy with $R=200$ : the value is very preliminary and only based on possible instrumentation/telescope design.

Table 1. Obervational capabilities.

\begin{tabular}{crrr}
\hline Band & Instrument/telescope & $\begin{array}{r}\text { point-source sensitivity } \\
(5-\sigma \text { in } 1 \text { hour })\end{array}$ & $\begin{array}{r}\text { distance limit } \\
(\mathrm{K}=-9)\end{array}$ \\
\hline $\mathrm{K}$ & SOFI/NTT & $20.9 \mathrm{mag}$ & $10 \mathrm{Mpc}$ \\
$\mathrm{K}$ & ISAAC/VLT & $22.0 \mathrm{mag}$ & $16 \mathrm{Mpc}$ \\
$\mathrm{K}$ & $\mathrm{NGST}$ & $26.0 \mathrm{mag}$ & $100 \mathrm{Mpc}$ \\
$\mathrm{N}$ & TIMMI2/3.6m & $2.5 \mathrm{mJy}$ & $1 \mathrm{Mpc}$ \\
$\mathrm{N}$ & $\mathrm{VISIR} / \mathrm{VTT}$ & $0.25 \mathrm{mJy}$ & $3 \mathrm{Mpc}$ \\
$\mathrm{Q}$ & $\mathrm{VISIR} / \mathrm{VLT}$ & $10: \mathrm{mJy}$ & $0.5 \mathrm{Mpc}$ \\
\hline
\end{tabular}

${ }^{a}$ Low-resolution spectroscopy

${ }^{b}$ Scaled from $F(12 \mu \mathrm{m})=1.0 \mathrm{Jy}$ at the LMC

\subsection{Individual stars}

The AGB population in the Magellanic Clouds is the only extra-galactic population for which extensive observations are available. ISO observations give the first reliable information on AGB mass loss and are presented elswhere in this volume (van Loon; Blommaert et al.).

AGB stars in more distant galaxies can be detected using infrared arrays, however this is so far limited to non-mass-losing stars. Davidge et al. (1998) present JHK images of the Sculptor group galaxies, and detect indications for luminous AGB stars below $K=16$, near the detection limit. Alonso et al. (1998) 
present VIJHK data for the dwarf galaxy NGC 3109. The AGB is clearly detected for ages down to $1 \mathrm{Gyr}$, but the survey is not deep enough to detect older AGB stars in the infrared. The new generation of wide-field infrared arrays is expected to give rapid progresses in the next few years.

Evolved AGB stars can also be identified through their variability, using I-band observations. Tolstoy et al. (1996) observed suspected Cepheid variables in $\mathrm{Gr}-8(\mathrm{~d}=2.4 \mathrm{Mpc})$. One star in their sample $(\mathrm{V}-1)$ both has a possible long period of 110 days and very red colours: it is presently the best candidate for a Mira-like variable outside the Local Group.

In the galactic dwarf spheroidals, the presence of carbon stars is indicative of intermediate-age populations (younger than globular clusters). Especially Fornax shows evidence for star formation less than $2 \mathrm{Gyr}$ ago (Beauchamp et al. 1995) and may well contain mass-losing stars. Whitelock et al. (1996) study carbon stars in the nearby Sagittarius dwarf: one star of their sample has since been shown to be a carbon Mira. A review of extra-galactic carbon stars is given by Groenewegen (these proceedings).

\subsection{Integrated properties}

Although individual mass-losing stars can only be detected at limited distances, their integrated flux over a galaxy may be appreciable. Knapp et al. (1992) studied elliptical galaxies in Virgo: they showed that on average 40 per cent of their $12-\mu \mathrm{m}$ emission may be due to circumstellar dust. The expected photospheric contribution is $S_{12}=0.08 S_{2.2}$ (with $S$ in Jy), whereas the observations for Virgo ellipticals give $S_{12}=0.138+/-0.014 S_{12}$. This is in contrast to globular clusters where the $12-\mu \mathrm{m}$ emission is consistent with purely photospheric emission (Knapp et al. 1995; Penny et al. 1997).

Recently, Bressan et al. (1998) have used the Padova isochrones to predict integrated IR colours of stellar populations for various mid-infrared bands. Their results rely heavily on assumed relations, e.g. between $Z$ and $\dot{M}$, but can easily be updated if required.

\section{Expansion velocities at low metallicity}

The expansion velocity $v_{\exp }$ of the gas is a result of the radiation pressure on the dust, and the 'drag' or coupling between the dust and the gas. This will depend on several parameters: $\dot{M}$ (or the optical depth through the envelope), the stellar luminosity $L$ and the dust-to-gas ratio. Habing et al. (1994) calculated outflow velocities as function of these parameters and showed that $v_{\text {exp }}$ reaches a maximum for intermediate values of $\dot{M}$ but continuously increases with $L$ : $v_{\text {exp }} \propto L^{0.35}$. However, a very large effect on the dust-to-gas ratio was found: the expansion velocities drop to only a few $\mathrm{km} / \mathrm{s}$ if the dust-to-gas ratio drops by a factor of 10 compared to AGB stars of solar metallicity, although the effect differs in magnitude between carbon and oxygen-rich stars.

Few observations of expansion velocities outside the galactic disk are available. Wood et al. (1992) determined $v_{\text {exp }}$ for five OH/IR stars in the LMC. They found values of only $60 \%$ of similar galactic OH/IR stars. This conclusion however depends on the way $v_{e x p}$ is measured: Wood et al. used the two strongest peaks in the $\mathrm{OH}$ spectrum which may not be appropriate for noisy 
spectra. If instead the outermost emission peaks are used, Zijlstra et al. (1996) find that the four confirmed AGB stars have $v_{\text {exp }}=12 \mathrm{~km} / \mathrm{s}$ versus the galactic comparison stars $v_{\text {exp }}=14 \mathrm{~km} / \mathrm{s}$, a difference of only $15 \%$. The fifth star of Wood et al. is a luminous supergiant: $\mathrm{SiO}$ emission showed that in this case the $\mathrm{OH}$ was not centred on the stellar velocity and therefore $v_{\exp }$ was greatly underestimated (van Loon et al. 1996).

Groenewegen et al. (1997) detected CO for one of the two known masslosing carbon-Miras in the galactic halo. The star has $v_{\text {exp }}=3.2 \pm 0.2 \mathrm{~km} / \mathrm{s}$. In comparison, galactic disk stars average around $10 \mathrm{~km} / \mathrm{s}$ (Groenewegen et al. 1998). A metallicity of $[\mathrm{Fe} / \mathrm{H}]=-0.7$ is estimated for the halo carbon star. The very low $v_{\text {exp }}$ is qualitatively consistent with the Habing et al. predictions, but further data on halo stars would be required for any conclusions.

The present data suggests that $v_{\text {exp }}$ may be lower at subsolar $Z$, but quantative information for a range of mass-loss rates and luminosities is lacking. The difference between the LMC and the Galaxy may be less than predicted by Habing et al. (1994), but this could easily be due to compensatory effects, such as higher luminosity and/or different pulsation properties in the LMC stars.

\section{Mass-loss rates at low metallicity}

The mass-loss formalisms mentioned in the Introduction do not include a direct $Z$-dependence. As the temperature on the early AGB is $Z$-dependent, this may indirectly introduce lower mass-loss rates at lower $Z$. (For the mass-losing part of the AGB we do not have data on how the temperature scale depends on $Z$.) On the other hand, the formalisms were not designed for different metallicities and cannot be used for predictions. If the mass loss is driven by radiation pressure on dust, a possibly quite large effect could be expected.

There are a number of observations of mass loss at low $Z$. Up to 50 masslosing AGB stars are now known in the LMC, with several having sufficiently high mass-loss rates to have significant extinction even at K. Zijlstra et al. (1996) conclude that for these stars, there is no evidence that $\dot{M}$ in the LMC is systematically lower than in the Galaxy. Also the SMC stars in their sample show no such effect. Groenewegen \& Blommaert (1998) find evidence for further highly obscured AGB stars in the SMC which also exhibit high mass-loss rates similar to those of galactic $\mathrm{OH} / \mathrm{IR}$ stars.

Further data comes from Mira variables in globular clusters and in the galactic halo. Groenewegen et al. (1998) derive dust mass-loss rates of a few $10^{-9} M_{\odot} y r^{-1}$ for two distant halo Miras with low Z. Mira variables in 47 Tuc and Omega Cen have been detected at $12 \mu \mathrm{m}$ and show similar mass-loss rates (Gillet et al. 1988; Origlia et al. 1995, 1997). The corresponding gas mass-loss rates are of order $10^{-6} M_{\odot} y r^{-1}$ (Origlia et al. derive much lower values but these are based on a Reimers' law. Their dust detection implies a much higher rate.) Frogel \& Elias (1988) find from a more complete sample an average $M>$ $10^{-7} M_{\odot} y r^{-1}$ for long-period variables in globular clusters with $[\mathrm{Fe} / \mathrm{H},>0.1$. Thus, variables in globular clusters show mass-loss rates within normal ranges. It should be noted that a correction may have to be made if the expansion velocities are very low. 
The data, within the limited accuracy, shows no evidence for low mass-loss rates at low $Z$. This may be related to the fact that an AGB star will continue to evolve to higher luminosities untill the mass-loss rate exceeds the nuclearburning rate. If mass loss is less efficient at low $Z$, the star will reach higher luminosity which will again increase the mass-loss rate (Habing et al. 1994). Thus, stars in different populations are likely to reach similar mass-loss rates, but may do so at different phases of their evolution (Bowen \& Willson 1991).

\section{Mira Period-Luminosity relations}

An important result obtained from extra-galactic AGB stars is the existence of a well-defined period-luminosity relation for Miras in the LMC. The relation was discovered by Glass \& Evans (1981). Feast et al. (1989) showed that the relation is especially narrow $(1-\sigma=0.13 \mathrm{mag}$.) when using the K-band. Not all Miras follow the $P L$ relation. LMC Miras with periods longer than 400 days tend to be overluminous (e.g. Zijlstra et al. 1996). In contrast, OH/IR stars near the Galactic Centre appear underluminous (Blommaert et al. 1998; Wood et al. 1998), although there are uncertainties in the bolometric corrections. A few nearby Miras are also underluminous (van Leeuwen et al. 1997).

Bedding \& Zijlstra (1998) show that nearby Miras and semi-regulars (SR) with accurate HIPPARCOS parallaxes define three sequences: one sequence agrees with the LMC Mira $P L$ relation, a broader sequence contains SR variables which is shifted by about a factor of two towards shorter periods (e.g. Wood \& Sebo 1996), and a few stars fall well below the relation. (A number of semiregulars show two periods, one of which agrees with the Mira relation and the other with the less narrow SR sequence.) The few stars below the relation have luminosities similar to the semiregulars (although having much longer periods) and may be evolutionary related to this group. The Miras show slightly higher luminosities and appear therefore more evolved. The luminosity difference between SR's and Miras is consistent with an evolution along a Whitelock (1986) track. From the HIPPARCOS data, life-times of the order of $510^{5} \mathrm{yr}$ can be estimated for the Mira phase and similar or larger for the SR's.

Wood (1990) predicts that the $P L$ relation should depend on $Z$, with galactic Miras being less luminous at $\mathrm{K}$ by 0.25 mag compared to LMC Miras of the same period (or, galactic Miras have longer periods). This is however not confirmed by available observations (e.g., van Leeuwen et al. 1997, Feast 1996, Whitelock et al. 1994). Alvarez et al. (1998) find some evidence for an offset between the HIPPARCOS Miras and the LMC PL relation, but the more accurate data of Bedding \& Zijlstra (1998) does not confirm this.

Fig. 1 compares the LMC Miras with HIPPARCOS Miras and with globular cluster Miras. The HIPPARCOS Miras (open circles) all have parallax uncertainties less than 15\%, and the resulting Lutz-Kelker bias is less than 0.05 mag (Koen 1992). A direct fit between the LMC and HIPPARCOS Miras (applying a bias correction according to Koen 1992) gives an LMC distance modulus of $18.63 \pm 0.09$, not inconsistent with other determinations. A shift of 0.25 mag would put the LMC at an unlikely distance of 18.9 and seems excluded.

Globular cluster Miras are shown in Fig. 1 as triangles. All occur in clusters with $[\mathrm{Fe} / \mathrm{H}]>0.1$ (Whitelock 1986). To scale the data, two Hori- 


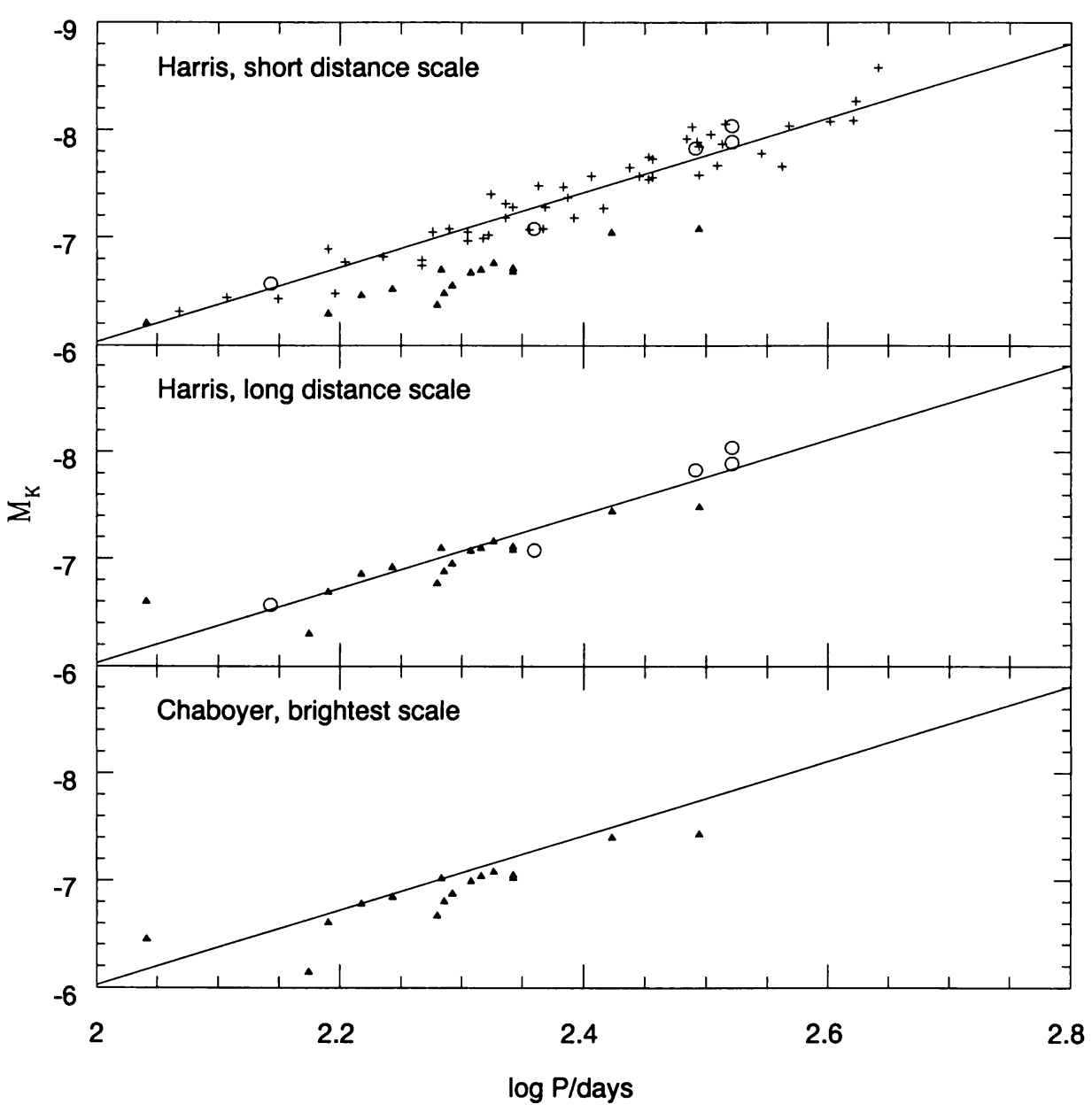

Figure 1. The Mira PL relations. Plus signs indicate LMC Miras (Feast et al. 1989), circles galactic HIPPARCOS Miras (Bedding \& Zijlstra 1998) and triangles Miras in globular clusters (Whitelock 1986). LMC Miras are scaled to a distance modulus of 18.57. Three different distance scales for globular clusters are shown, based on different calibrations for the horizontal branch magnitude. 
zontal Branch distance scales of Harris (1996) are used, a short scale (derived from RR Lyrae statistical parallaxes) and a long one (field subdwarfs). The average scale corresponds to $M_{V}(\mathrm{HB})=0.15[\mathrm{Fe} / \mathrm{H}]+0.85$. The bottom panel uses the Chaboyer (1998) relation $M_{V}(H B)=0.23([\mathrm{Fe} / \mathrm{H}]+1.6)+0.46$ which again is $0.1 \mathrm{mag}$. brighter than an average including the statistical parallax. It is clear that only the brightest scale gives good agreement with the Mira $P L$ relation. A direct fit to the $L M C$ data using the Harris scale gives $M_{V}(H B)=0.15[F e / H]+0.60 \pm 0.03$. (This would yield globular cluster ages of $11+/-2$ Gyr.)

There is thus little evidence for a dependence of the $P L$ relation on $Z$, when using K-band magnitudes. If the relation is indeed invariant, it would make Mira variables a highly useful distance indicator.

\section{Mass loss and the PL relation}

The position of a Mira in the $P L$ diagram is determined by the core-massluminosity relation and by the pulsation equation:

$$
\log P=1.5 \log R-0.5 \log M+\log Q
$$

where $P$ is the period in days, $R$ and $M$ are the radius and mass in solar units and $Q$ is the pulsation constant. Using the AGB relation of Wood (1990):

$$
M_{\text {bol }}=15.7 \log T_{\text {eff }}+1.884 \log z-2.65 \log M-59.1-15.7 \Delta
$$

(where the disturbance term $\Delta$ is usually ignored), one finds (Feast 1996):

$$
M_{\text {bol }}=-2.036 \log P+0.73 \log z-2.049 \log M+2.881+2.036 \log Q
$$

Here $z=Z / Z_{\odot}$. The existence of a narrow $P L$ relation implies $P$ is not an independent parameter: $M_{\text {bol }}=f(z, M)$. Note that $z$ is not $a$ priori known for an LMC star but may vary between stars of different ages. $M$ is not constant, due to mass loss. In addition, $M_{\mathrm{bol}}$ is determined by the core mass independently from $M$, but will change by as much as $1 \mathrm{mag}$. over the phase of the thermalpulse cycle. These separate effects should lead to a large broadening of the $P L$ relation (e.g. Vassiliadis \& Wood 1993). From the narrow $P L$ relation we find:

$$
d P / d M=0 ; \quad d \log P / d M_{\text {bol }}=-0.33 ; \quad d P / d z=0,
$$

valid during the mass loss phase. (The second relation is the observed $P L$ relation.) To keep a star on the $P L$ relation during the Mira phase, the stellar radius must adjust to compensate for the changing luminosity and mass. The AGB relation above, valid for the earlier AGB, may thus not fully describe Miras.

The observed radius is wavelength dependent, due to molecular bands in the extended atmosphere. This re-introduces a metallicity effect, seen as a colour term dependent on $z$ (Feast 1996). The $P L$ relation is best defined at $\mathrm{K}$, where the atmospheric bands do not contribute and the observed radius is closest to the photospheric radius. 

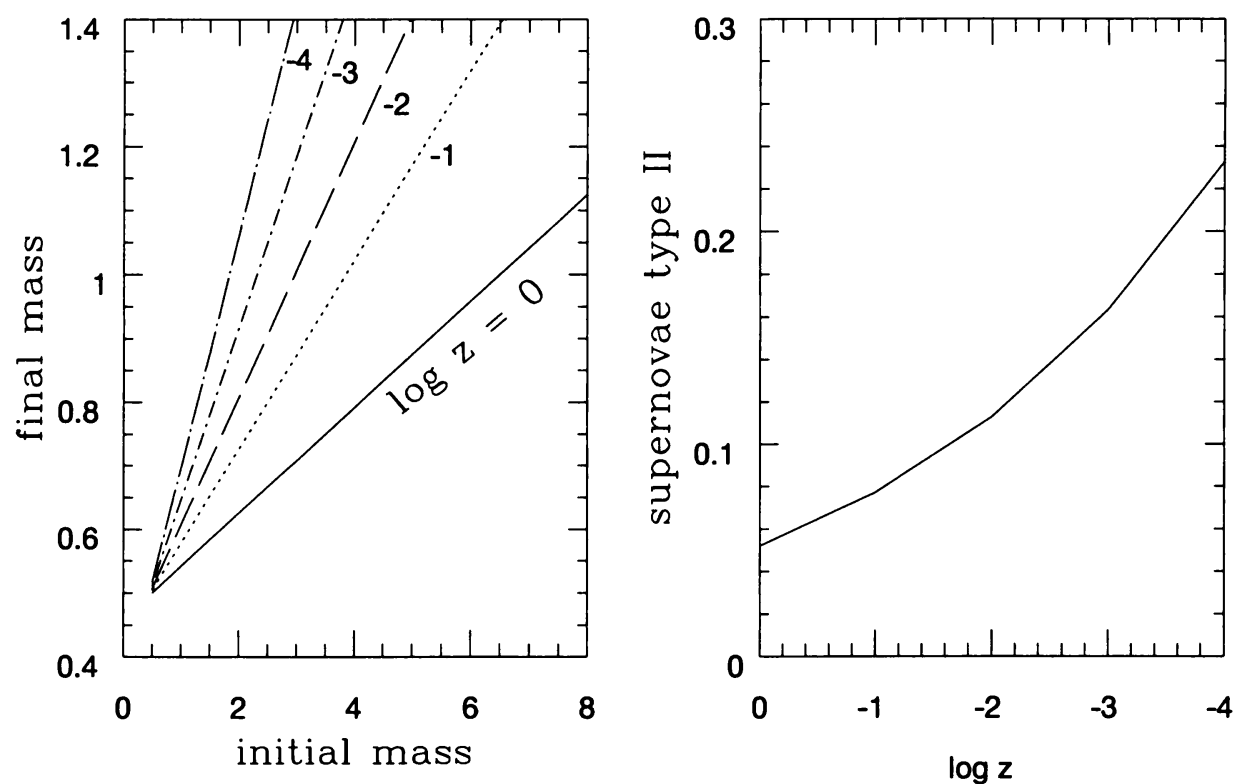

Figure 2. Left: initial-final mass relations for intermediate-mass stars at low $z$. Right: The inferred relative number of type-II (postmain-sequence) supernovae as function of $\log z$, integrated over the first Gyr.

A possible explanation for the relations above is a feedback between the stellar pulsations and mass loss. Such a mechanism could break down either at high luminosities or at high mass-loss rates. The latter case could correspond to the underluminous stars at the Galactic Centre.

\section{Initial-Final Mass relations at low $\mathrm{Z}$}

The AGB mass-loss formalisms include an $R$-dependence. Using the $R \propto Z^{0.088}$ relation of Iben (1984) for the AGB predicts that a low- $z$ AGB star will have a slightly lower $\dot{M}$ at the same luminosity. For $z=0.1$, the Reimers mass loss would be $20 \%$ lower. To compensate, a low- $z$ star would require a higher luminosity (and therefore a higher core mass) to reach the same $\dot{M}$. Assuming the evolution is terminated when $\dot{M}$ exceeds the nuclear burning rate, a low- $z$ star is expected to reach a higher final core mass and thus yield a more massive white dwarf than a star with the same initial mass but higher $z$.

For $z<0.1$, the effect of radiation pressure of dust becomes very small and the mass loss is probably purely driven by the stellar pulsation. For such a dustless wind, Bowen \& Willson (1991) find that $M_{\text {bol }}$ should be 0.3 mag. brighter to reach the same mass loss as a dusty wind. The total increase in brightness as function of $Z$ to reach the same mass-loss rates is $-\Delta M_{\mathrm{bol}}=$ $0.30-0.33 \log z$, for $z=Z / Z_{\odot}<0.1$. 
The core mass-luminosity relation $L=59250\left(M_{c}-0.495\right)$ (e.g. Boothroyd \& Sackman 1988) can be used to quantify the effect on the final mass of the resulting white dwarf. The required increase is very small for low core mass, but becomes significant for core masses corresponding to the heaviest white dwarfs. We use an approximate present initial-final mass relation of $M_{f}=$ $0.5+M_{i} / 12$, and a Salpeter IMF $d N / d M=m^{-2.35}$. Fig. 2 shows the resulting $M_{i}-M_{f}$ relation as function of $z$. At $z<0.01$, the heaviest remnants reach the Chandresekhar mass giving rise to a class of AGB supernovae. At the extreme $z$, the contribution of these AGB-SN exceeds the type II SN by a factor of a few, over the first Gyr of the stellar population. If confirmed, such an effect would have dramatic consequences for the evolution of the earliest stellar populations in the high-redshift Universe.

\section{References}

Alonso V.M., Minniti D., Zijlstra A.A., Tolstoy E., 1998, A\&A, submitted

Alvarez R., Mennessier M.-O., Barthès D., Luri X., Mattei J.A., 1997, A\&A 327,656

Beauchamp D., Hardy E., Suntzeff N.B., Zinn R., 1995 AJ 109, 1628

Bedding T.R., Zijlstra A.A., 1998, ApJ 506, L47

Blommaert J.A.D.L., van der Veen W.E.C.J., van Langevelde H.J., Habing H.J., Sjouwerman L.O., 1998, A\&A 329, 991

Boothroyd A., Sackman I.-J., 1988, ApJ 328, 641

Bowen G.H., Willson L.A., 1991, ApJ 375, L53

Bressan A., Granato G.L., Silva L., 1998, A\&A 332, 15

Chaboyer B., 1998, http://xxx.lanl.gov/abs/astro-ph/9808202

Davidge T.J., 1998, ApJ 497, 650

Feast M.W., 1996, MNRAS 278, 11

Feast M.W., Glass I.S., Whitelock P.A., Catchpole R.M., 1989, MNRAS 241, 375

Frogel J.A., Elias J.H., 1988, ApJ 324, 822

Gillet F.G., de Jong T., Neugebauer G., Rice W.L., Emerson J.P., 1988, AJ 96, 116

Glass I.S., Evans T.L., 1981, Nature 291, 303

Groenewegen M.A.T., Whitelock P.A., Smith C.H., Kerschbaum F., 1998, MNRAS 293, 18

Groenewegen M.A.T., Blommaert J.A.D.L., 1998, A\&A 332, 25

Groenewegen M.A.T., Oudmaijer R.D., Ludwig H.-G.,1997, MNRAS 292, 686

Habing H.J., Tignon T., Tielens A.G.G.M., 1994, A\&A 286, 523

Harris W.E., 1996, AJ 112, 1487 (http://www.physics.mcmaster.ca/Globular.html)

Iben I., Jr., 1984, ApJ 277, 333

Koen C., 1992, MNRAS 256, 65

Knapp G.R., Gunn J.E., Wynn-Williams C.G., 1992, ApJ 399, 76 
Knapp G.R., Gunn J.E., Connolly A.J., 1995, ApJ 448, 195

Origlia L., Ferraro F.R., Fusi Pecci F., 1995, MNRAS 277, 125

Origlia L., Scaltriti F., Anderlucci E., Ferraro F.R., Fusi Pecci F., 1997, MNRAS 292, 753

Reid I.N., 1991, ApJ 382, 143

Tolstoy E., Daha A., Hoessel J.G., Danielson G.E., 1996, AJ 109, 579

van Leeuwen F., Feast M.W., Whitelock P.A., Yudin B., 1997, MNRAS 287, 955 van Loon J.Th., Zijlstra A.A., Whitelock P.A., te Lintel Hekkert P., Chapman J.M., Loup C., Groenewegen M.A.T., Waters L.B.F.M., Trams N.R., 1998, A\&A 329, 169

van Loon J.Th., Zijlstra A.A., Whitelock P.A., Waters L.B.F.M., Loup C., Trams N.R., 1997, A\&A 325, 585

van Loon J.Th., Zijlstra A.A., Bujarrabal V., Nyman L.-A., 1996, A\&A 390, L29

Vassiliadis E., Wood P.R., 1993, ApJ 413, 641

Whitelock P.A., 1986, MNRAS 219, 525

Whitelock P.A., Menzies J.W., Feast M.W., Marang F., Carter B.S., Roberts G., Catchpole R.M., Chapman J.W., 1994, MNRAS 267, 711

Whitelock P.A., Irwin M., Catchpole R.M., 1996, New Astronomy 1, 57

Wood P.R., 1990, in From Miras to Planetary Nebulae: Which path for stellar evolution?, M.-O. Mennessier \& A. Omont (eds.), Editions Frontières, p. 32

Wood P.R., Habing H.J., MacGregor P.J., 1998, A\&A 336, 925

Wood P.R., Whiteoak J.B., Hughes M.B., Bessell M.S., Gardner F.F., Hyland A.R., 1992, ApJ 397, 552

Wood P.R., Sebo K.M., 1996, MNRAS 282, 958

Zijlstra A.A., Loup C., Waters L.B.F.M., Whitelock P.A., van Loon J.Th., Guglielmo F., 1996, MNRAS 279, 32 